\title{
Silicon Photomultiplier for the Plug \& Imaging PET System: Physics, Technological Challenges and Application to Modern Nuclear Medicine
}

\author{
Nicola D'Ascenzo and Qingguo Xie \\ Additional information is available at the end of the chapter \\ http://dx.doi.org/10.5772/intechopen.73007
}

\begin{abstract}
We propose the design of a Silicon Photomultiplier at the $180 \mathrm{~nm}$ GLOBALFOUNDRIES BCDLITE CMOS technology node. We perform a characterization of the device, in comparison with other results obtained a CMOS technology node and we investigate the limits and strengths of this approach. Finally we show possible future applications of the $\mathrm{SiPM}$ in Nuclear Medicine, in particular to digital positron emission tomography systems.
\end{abstract}

Keywords: silicon photomultiplier, avalanche breakdown, positron emission tomography

\section{Introduction}

Low photon flux sensors are specific devices dedicated to the detection of a small number of photons. The classical example of such sensors is the photomultiplier tube (PMT), which was dominating the field during the last 70 years. PMT were broadly applied to high energy physics, nuclear medicine, space applications, among others, despite their complicated manufactory which includes vacuum technology, high bias voltage up to few thousand volts, large and not scalable size [1].

The advances in semiconductor technology during the last 20 years were beneficial for the development of modern light wave and telecommunication systems. The avalanche photodiode (APD) is currently one of the most spread semiconductor-based photo-detectors for low photon flux. APD allowed to study fundamental properties of the light in order to improve specific applications in photonics, including quantum coherence of multi-photon states and decoherence among others $[2,3]$. 
PMT, APD and low photon flux sensors derived from them have a strong limitation. Their sensitivity to the detection of a single photon is affected by a large statistical fluctuation in the signal generation and do not allow de facto an efficient disentangling of single photon states.

In the late 1970s a new sensor, later known as silicon photomultiplier (SiPM), was invented [4]. It consisted of a space-distributed fine array of metal resistor semiconductor (MRS) microsensors with individual quenching and common output. Since then, a large number of groups contributed in the development of the modern SiPM, which is nowadays commercially available and started to substitute the PMT in the above-mentioned application fields [4-12].

Modern SiPMs are composed of an array of $\mathrm{p} / \mathrm{n}$ junctions (microcell) operated in Geiger mode. A single photon interacts with a microcell through the photoelectric effect and generates an electron/hole pair, which initiates a self-sustaining avalanche. The quenching is realized passively with an integrated resistor in the microcell.

In parallel the similar technology of the single photon avalanche diode (SPAD) was developed [13]. The SPAD is a single photon detector operated in Geiger mode. Its optical sensing part is equivalent to a microcell of the SiPM. In addition the layout of a SPAD includes integrated active quenching electronics components [14]. In other words the SiPM is equivalent to an array of SPAD-like microcells with passive quenching.

The current advance in the development of SiPM and SPAD is represented by the complementary metal oxide semiconductor (CMOS) technology. The implementation of SiPM and SPAD sensitive cells in standard CMOS technology allows the combined integration of sensor and read-out electronics on the same device, with a significant reduction of power consumption and simplification of the operational conditions. In recent years a large number of advanced digital imagers and innovative concepts for light detection were proposed on this basis [15-18].

However some drawbacks of the application of the CMOS technology to photonics sensors operated in avalanche breakdown mode were identified in the last few years. CMOS processes introduce a higher level of noise and lower photon detection efficiency, with respect to customtechnologies [19-53].

Among the many fields of applications, photonics sensors realized in CMOS technology are expected to have a strong impact in Nuclear Medicine, and it is maybe better to introduce here this concept with a certain detail. Nuclear Medicine is dealing with the diagnosis and treatment of patients with ionizing radiation. From the diagnostic point of view computed tomography (CT) and positron emitted tomography (PET) machines are nowadays conventionally used in hospitals for the detection of cancer lesions among others [54, 55]. From the clinical point of view proton therapy is nowadays being used in various centers for a more effective cure of cancer with respect to chemotherapy.

In this chapter we provide a description of the technological challenges in the development of modern SiPM in CMOS technology. We base our discussion on two SiPM structures manufactured at the BCDLITE $180 \mathrm{~nm}$ GF CMOS technology node. We perform a characterization of the devices in dark and light illumination conditions, putting the results in the broader context of other structures realized in CMOS and in custom technology. Finally we provide an application of such optical sensors to Nuclear Medicine, showing how such 
small-sized novel photo-detectors have an impact in the technology for positron emission tomography (PET) machines in the new digital generation.

\section{Physical principles of the SiPM}

The basic component of a SiPM is a sensitive microcell. A simplified SiPM structure is shown on Figure 1. Within a n-doped region (nwell), a heavily p-doped implantation is formed $\left(\mathrm{p}^{+}\right)$, generating a $\mathrm{p}+/$ nwell diode. The general schematics proposed here is not applicable to any working devices, due to many technological and operational constrains. However, this simplified layout of the microcell is used here in order to describe the physical background of the device. Several examples of real implementations of such device will be shown in the next section.

The SiPM is composed of an array of such microcells. The typical microcell pitch ranges between 20 and $100 \mu \mathrm{m}$. In analog SiPMs the microcells have common anode and cathode as reported in Figure 2. In digital SiPMs each microcell is read-out separately with a dedicated digital electronic processing as reported in Figure 3.

When the microcell is reverse-biased, a depletion region is formed between the $p+$ and the nwell implantations. The typical depth of the depletion region is approximately $800 \mathrm{~nm}$ from the microcell surface and the typical width is approximately $1 \mu \mathrm{m}$. The size and the concentration of the depletion region defines also the capacitance of a single microcell, which reaches

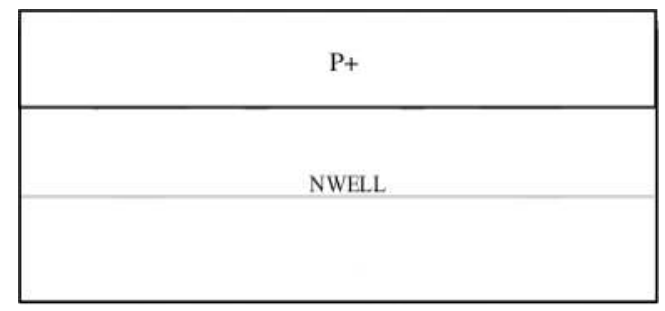

Figure 1. General simplified cross-section of a silicon photomultiplier microcell.

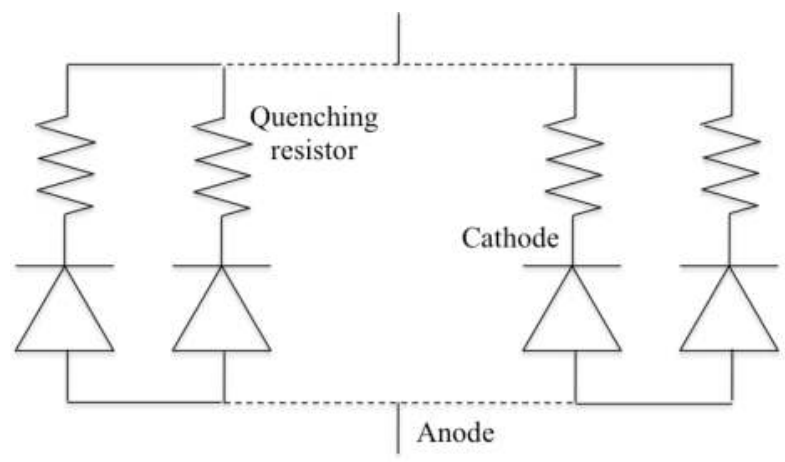

Figure 2. Schematics of an analog silicon photomultiplier. 

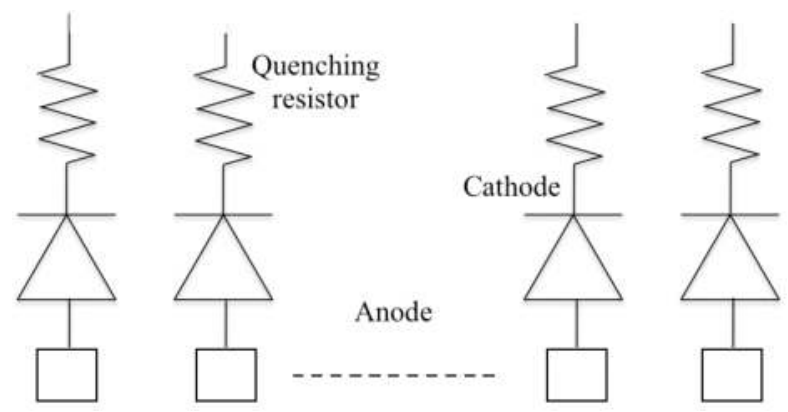

Figure 3. Schematics of a digital silicon photomultiplier with a dedicated read-out digital electronics for each microcell.

few hundreds fF. The reverse-biased microcell exhibits an electric field with maximal strength ranging between $10^{5}$ and $10^{6} \mathrm{~V} / \mathrm{cm}$. The strength of the electric field as a function of the total charge density is governed by the Poisson Eq.

A typical feature of the SiPM as quantum detector is its bi-stability, i.e. its operation allows two stability states with a continuous transition between them. The two states correspond to the physical processes of carrier recombination and generation occurring in the $\mathrm{p}+/$ nwell junction in the operational condition of the SiPM.

The state I of the SiPM microcell is determined by the electron-hole recombination mechanism. Electrons are dropping from the conduction to the valence band, transferring their excess energy to other electrons, phonons or photons. The involved physical processes can be radiative and

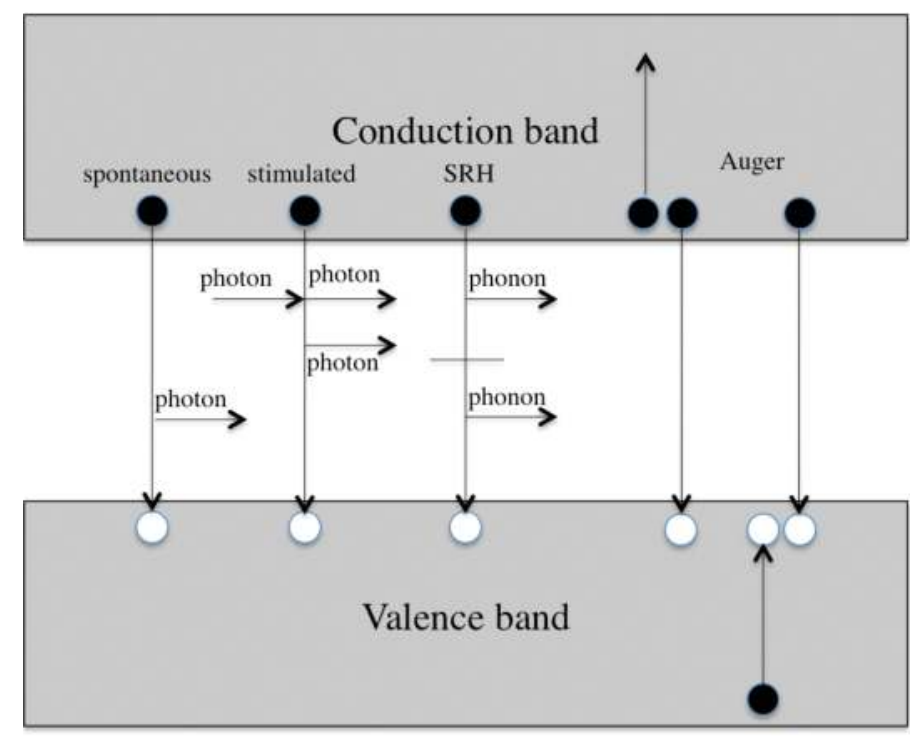

Figure 4. Radiative and non-radiative recombination processes in semiconductor devices. 
non-radiative and are depicted in Figure 4. In the first case a photon is emitted, in the second case the energy is transferred only to phonons or electrons. The generation of photons by radiative recombination can be spontaneous or stimulated by an additional photon. The two main nonradiative processes are Shockley-Read Hall (SRH) recombination and Auger recombination [56]. $\mathrm{SRH}$ involves energy levels generated by crystal defects. Such energy levels capture electrons from the conduction band as well as holes from the valence band. The excess energy is released through phonons. In Auger recombination the excess energy is transferred to other electrons within the valence and the conduction band. A main result of the recombination process is that electrons, photons and phonons are released, which can either initiate further recombination process or generate electron/hole pairs. Because of the properties of the Fermi distribution function of carriers in silicon, the process of electron-holes generation through recombination is temperature-dependent and is often called thermal generation of electron/hole pairs.

The state II of the SiPM microcell is determined by the impact ionization process [57]. The impact ionization is the reverse Auger process as the energy of motion of an electron is absorbed in order to produce new electron/hole pairs. At the strong electric field inside the SiPM microcell, both electrons and holes participate to the charge multiplication process. In this condition an infinite number of charged carriers is produced by a single electron/hole pair. In other words the avalanche induced in the state II is self-sustaining.

In normal conditions the recombination process would bring the junction back to the equilibrium of the doping concentration, in the SiPM the produced electron/hole pairs are accelerated in the strong electric field and start a multiplication chain due to impact ionization. The transition between state I and state II is as fast as 100 ps. The transition back from state II to state I requires a feedback process. A passive quenching resistor is placed in series with a microcell. The current flow through the resistor causes a voltage drop on the junction, which reduces the actual junction bias to a value lower than the breakdown voltage. After the quenching occurs, the SiPM transits back to the state I within a recovery time determined by the size of the junction capacitance and of the quenching resistor. The typical value of the quenching resistor is between $100 \mathrm{k} \Omega$ and $1 \mathrm{M} \Omega$.

Besides the thermal process, photon detection triggers the transition between the state I and the state II. When an optical photon is detected, an electron/hole pair is generated through photoelectric effect. Such e/h pair initiates the avalanche process through impact ionization.

A typical signal corresponding to the transition between the states I and II is shown on Figure 5. The current flowing through a single microcell is shown. At the beginning the microcell is in state I, with a low current of few picoamperes. A thermally generated pair causes the transition to the state II, which determines a fast rise of the signal with a peak at $30 \mu \mathrm{A}$, where the quenching time is reached. After that, within an exponential decay time governed by the quenching resistor and the intrinsic capacitance of the junction, the current reaches again the state I. The signal corresponding to a value of the quenching resistor 250 and $300 \mathrm{kOhm}$ is reported. We observe that the only effect introduced by the change of quenching resistor consists of a larger decay time, being the total charge under the pulse constant. The total charge contained in the signal is corresponding to approximately $1.5 \times 10^{6}$ electrons. Thus from a single electron/hole pair produced in the sensitive area of the device, a signal corresponding to this large number of electrons is produced. 


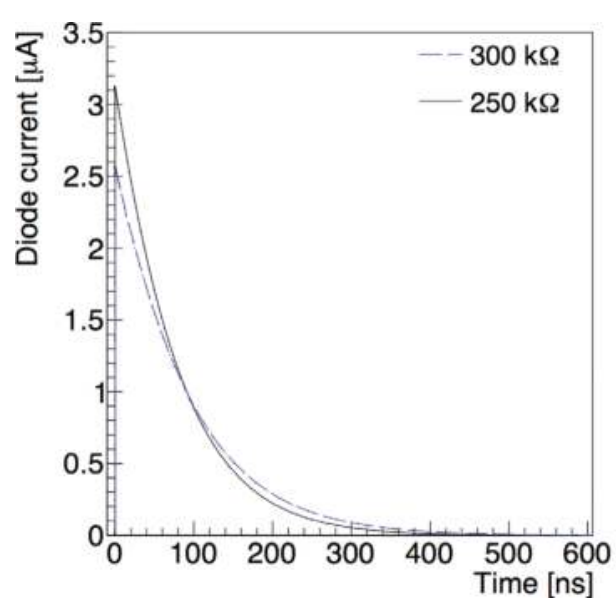

Figure 5. Typical diode current pulse corresponding to a thermally generated electron/hole pair. The transition between state I and state II is observed for two different quenching resistors.

The SiPM is hence a device which is sensitive to the energy deposited by a single visible photon and is able to produce a sizeable signal in correspondence to such energy. The signal is produced through a combination of quantum effects and a transition from the two stability states within a time of few tens picoseconds. Due to the ability detection of a single quantum of light and to the quantum working principle of the device itself, the SiPM is called sometimes "quantum detector".

\section{SiPM in CMOS: challenges and limitations}

The development of SiPM in the CMOS framework faces non-trivial issues. As we described in the previous section, SiPM is based on $\mathrm{p}+/ \mathrm{n}$ or $\mathrm{n}+/ \mathrm{p}$ junction structures working in two states defined by recombination and generation processes. Although the structure proposed in the previous section has a strong physical meaning, its realistic manufacturing in existing technology encounters several difficulties.

The most relevant problem in the integration of such structures within CMOS technology is that they can suffer of localized breakdown conditions on the locally concentrated high electric field at the junction edges. The use of guard ring structures around the sensitive area of each microcell is in this respect mandatory in order to obtain a uniform electric field across the whole sensitive area. Although CMOS compatible technology offers some possibility of implementing such guard rings [58-61], it is normally needed to add specific masks in order to allow the overlap between highly-doped and low-doped regions and to correct the doping profiles.

A second problem in the development of SiPM quantum devices within standard CMOS technology is the difficulty to control the thermal e/h pairs generating the dark noise of the 


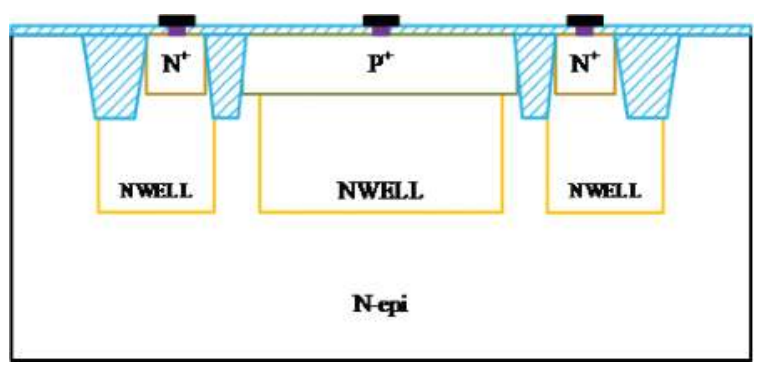

Figure 6. The concept of virtual guard rings.

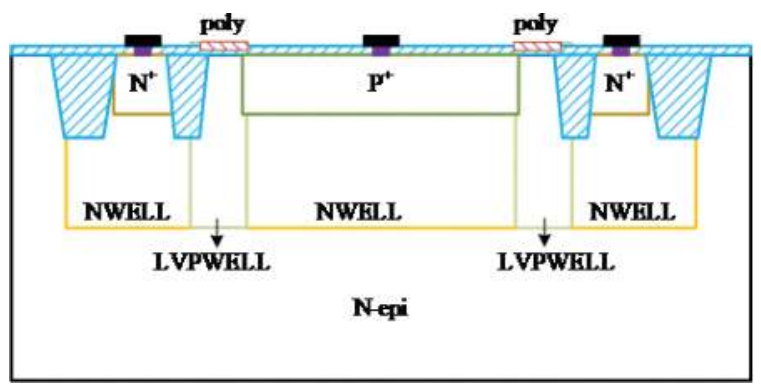

Figure 7. The concept of diffused lowly doped p-type guard rings.

1optical detector. The thermal e/h production rate depends on the concentration of the $\mathrm{n}$ - or $\mathrm{p}$ well and on the impurities introduced in the production stage. There is a current trend to design APIX sensors within modern deep-sub-micron CMOS processes, below the $180 \mathrm{~nm}$ scale. In this case the increased concentration of the wells, needed to enable the reduced design rules, causes a lowering of the breakdown voltage down to approximately $10 \mathrm{~V}$, with a significant increase of thermally generated e/h pairs due to band-to-band tunneling effect. Moreover, as we depicted in the previous section, defects, contributing to the thermally generated e/h pairs through SRH recombination, are of larger number in such modern CMOS technologies, due to lower annealing temperatures and to the presence of the shallow trench isolations (STI) close to the sensitive region of the optic detector.

A third challenge of the development of SiPM quantum detectors into standard CMOS is the correct design of the optical coupling, needed to optimize the photon detection efficiency at a visible wavelength. The production lines of standard commercial CMOS fabs are mainly oriented to large-scale market applications, as standard electronics components for automotive and energy industry among others. Although the optical communication industry plays a fundamental role in the actual panorama, it uses high power standard light sources, which are affected by low power losses and have different needs in comparison to weak visible light. Low photon flux sensors in the optical range occupy clearly a smaller market section and most of the standard CMOS technology fabs do not provide the necessary anti reflective coating (ARC). 
The most severe limitation of CMOS processes for the design of the SiPM is that a series of design rules needs to be verified. In CMOS technology there is an enclosure hierarchy between active area, $\mathrm{p}+$ implantation and nwell. A minimal distance between $\mathrm{p}+$ implantation and pwell should be also preserved as well as between active area and polysilicon resistance elements. As an example at a scale $\lambda=180 \mathrm{~nm}$ CMOS the minimal distance between pwell implantations is approximately $2 \lambda$ and the enclosure of $p+$ implantation and active area is approximately $1 \lambda$. It is often happening that one is forced to violate some of these rules in order to produce a Silicon Photomultiplier device.

Several studies were performed at a CMOS scale ranging between 90 and $800 \mathrm{~nm}$ [19-55]. A possible implementation solution consists of designing diffusive guard rings, as shown on Figure 6. As an example such layout was developed using the Teledyne DALSA $800 \mathrm{~nm}$ HV and the BCDLITE GLOBALFOUNDRIES $180 \mathrm{~nm}$ CMOS processes [18, 55]. Such layout is based on an inverted enclosure of the $\mathrm{p}+$ and nwell implantations, so that the high electric field zones at the edges of the diode are corrected with the geometrical distribution of the carriers. In this case only the CMOS enclosure rule is violated. Another possibility is offered by the implementation of diffusive lowly doped $\mathrm{p}$ guard rings around the $\mathrm{p}+$ implantation, as depicted in Figure 7. Such layout was developed using the Teledyne DALSA $800 \mathrm{~nm}$ HV, $350 \mathrm{~nm}$ HV AMS and Chartered GLOBALFOUNDRIES $130 \mathrm{~nm} /$ Tezzaron CMOS technology $[18,37,41]$. In this case the lowly doped region is smoothing the electric field at the edges of the junction.

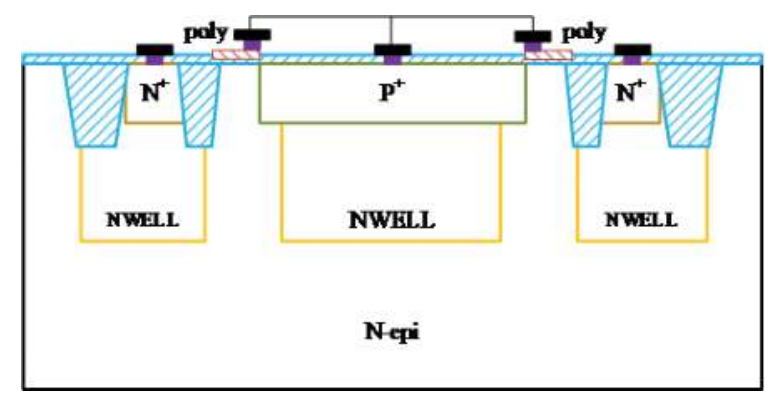

Figure 8. Structure I: a virtual guard ring with corrected STI.

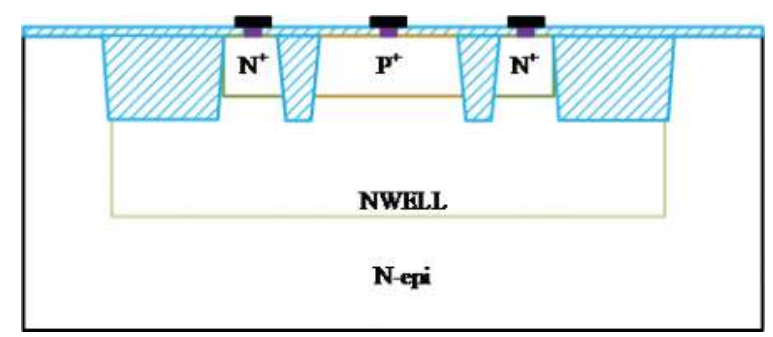

Figure 9. Structure II: a STI guard ring. 
One way to prevent the violation of design rules is to include additional masks in the CMOS process, i.e. implementing new design rules accommodating the needs of the sensor, without a perturbation of the transistor development. As an example in $[15,17]$ an additional mask is implemented in order to produce the p-diffusive guard ring. Another way consists of finding innovative approaches for controlling the properties of the diode edge. In [28, 29] a layout is proposed with perimeter-gated guard rings. In this case a $\mathrm{p}+/$ nwell diode is surrounded by a gate. A gate bias ranging from 0 to $8 \mathrm{~V}$ is applied to the structure in order to shape the electric field at the edges of the diode and avoid localized breakdown.

Following these design ideas and suggestions, we present here two possible design solutions obtained at the BCDLITE LV GLOBALFOUNDRIES $180 \mathrm{~nm}$ CMOS node. Structure I on Figure 8 is based on an n-epitaxial layer on which the SiPM sensor is formed. The microcell consists of a $50 \times 50 \mu \mathrm{m} n+/$ pwell junction. GF does not share the exact doping of the wells with the customers. A virtual guard ring $\mathrm{p}+$ /nepi is formed on the periphery of the sensitive avalanche area. As a difference from [55], a polysilicon ring is placed around the p+ implantation in order to prevent the formation of STI close to the sensitive area. Structure II on Figure 9 is based on an n-epitaxial layer on which the SiPM sensor is formed. The microcell consists of a $50 \times 50 \mu \mathrm{m} \mathrm{n}+/$ pwell junction. STI is used around the sensitive area as guard rings. The aim of the study of these two structures is to verify up to which amount STI are deteriorating the performances of the SiPM.

Two series of measurements have been carried out for the SiPM sensor prototypes proposed in this chapter: a static and a dynamic characterization. In this section we show only the characterization in dark condition and we postpone the characterization of the light response to the next section. The benchmarking of the experimental results needs to follow a twofold approach. On the one side it is needed to benchmark against the mature available SiPMs, which are not always developed in standard CMOS technology but in dedicated lines with additional optimized masks. On the other side it is needed to benchmark against the abovementioned experimental results, which are based on other CMOS processes. This second comparison allows us to understand how the chosen CMOS technology process performs with respect to other available ones.

The measurement of the current-voltage characteristic is performed on the single microcell test structure at wafer level. A Keithley 2636A source meter, connected to a computer, obtains measurements of current in reverse mode. This experiment is achieved by generating a sweep voltage between 0 and $25 \mathrm{~V}$, then measuring the current and limiting it to $20 \mathrm{uA}$ in order to avoid damaging the device.

The current-voltage curve of the first structure in reverse mode is shown on Figure 10. The structure exhibits a dark current below a few picoamperes before avalanche breakdown. At breakdown the current rises abruptly up to a few microamperes. After breakdown it gets limited by the quenching resistor and rises linearly. The breakdown voltage is approximately 13.2 V. On the plot few curves corresponding to measurements performed on chips obtained in different wafers are shown.

The current-voltage curve of the second structure in reverse mode is shown in Figure 11. The structure exhibits a dark current below a few picoamperes before avalanche breakdown. 


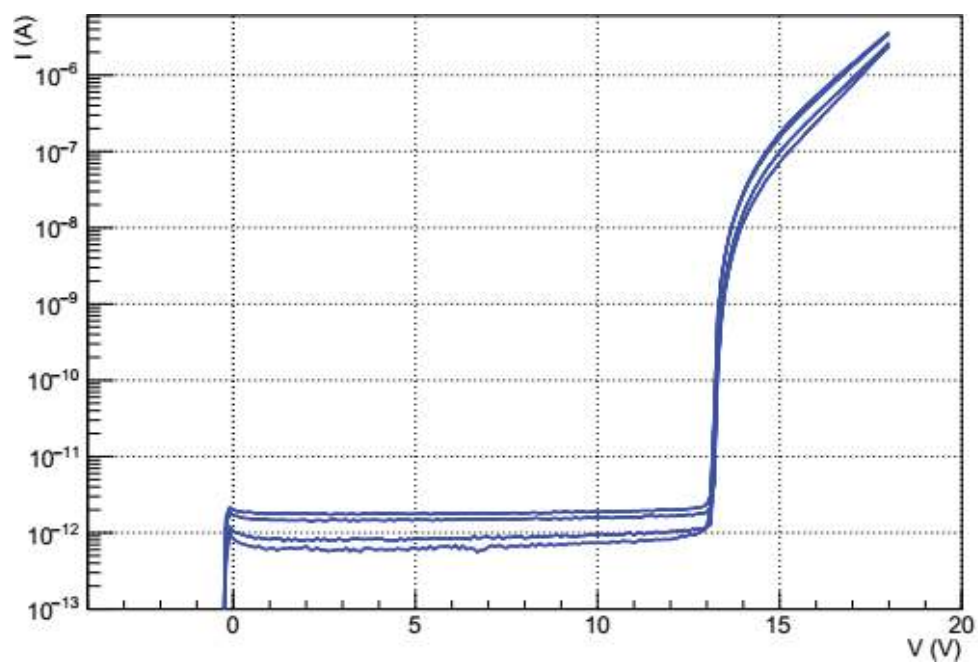

Figure 10. Current-voltage characteristics of structure I.

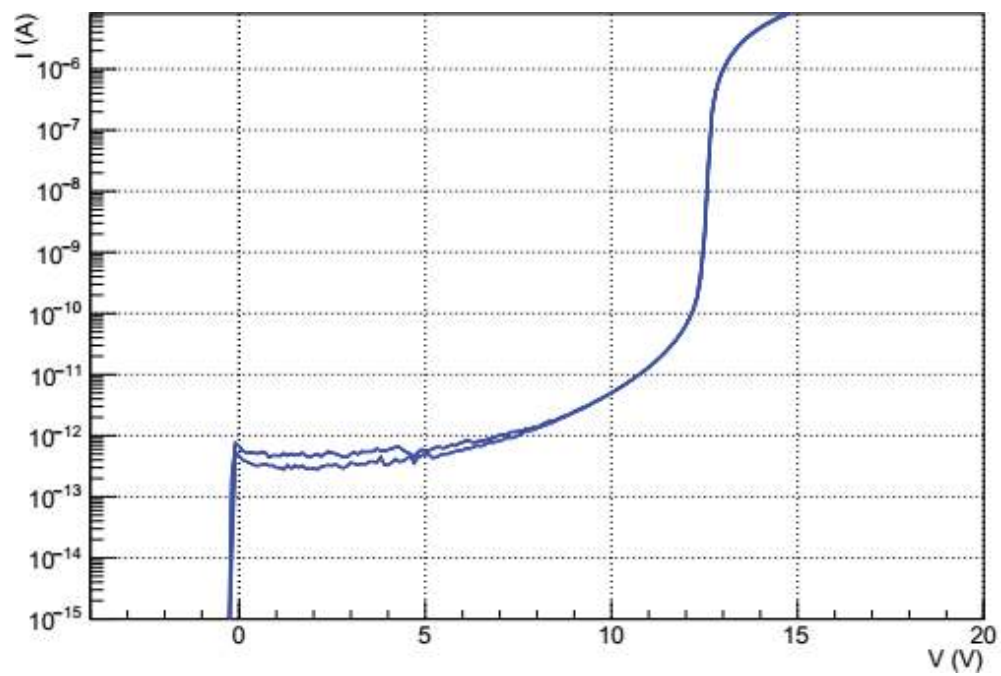

Figure 11. Current-voltage characteristics of structure II.

At breakdown the current rises up to a few microamperes. After breakdown it gets limited by the quenching resistor and rises linearly. The breakdown voltage is approximately $12.4 \mathrm{~V}$. In comparison with the previous structure we notice here an additional noise source, which affects the result. Such noise source is due to the leakage at the sides of the STI. On the plot few curves corresponding to measurements performed on chips obtained in different wafers are shown. 
The additional noise introduced by the STI has an impact also on the dynamic performance in dark conditions. The dynamic characterization consists of detecting the transition signal from the state I to the state II, which is normally called dark pulse. The verification of this signal and the measurement of its occurrence rate give an impression of the purity of the device. In fact, as the measurement is done in dark conditions and on a single microcell, the quality of the signal and its rate are directly proportional to the amount of impurities present in the device, which are triggering the thermal emission of electron/hole pairs trough SRH mechanism.. We measure the dark count rate by counting the number of avalanche signals produced in the SiPM sensor operated $2 \mathrm{~V}$ above breakdown. The dark rate is measured at room temperature and in dark conditions. The voltage amplitude of the signal of the SiPM is measured on a $50 \mathrm{Ohm}$ load resistor. The output voltage is connected to a fast amplifier based on a two-stage voltage amplifier obtained with the Gali 5+ wide-band monolithic chip [62]. The total amplification gain is adjusted to 10 with a voltage divider between the two amplification stages. The signal is sent to a threshold discriminator (CAEN N844). The number of pulses above threshold are registered within a $1 \mathrm{~s}$ observation time window.

The signals observed at the oscilloscope within a $1 \mu$ s time window are shown on Figures 12 and 13. We observe that the structure I presents a clear signal corresponding to the production of a thermal electron/hole pair. The signal has a typical rise time of few hundred picoseconds and a decay time of $67 \mathrm{~ns}$. The timing property is consistent with a quenching resistor of 250 $\mathrm{kOhm}$ and a capacitance of approximately $300 \mathrm{fF}$. Structure II shows a similar timing property. However dark pulses are occurring with higher frequency. This effect is due to the additional leakage coming from the STI walls. The dark rate measured at a overvoltage of $1.5 \mathrm{~V}$ at an amplitude of 0.5 dark pulse is respectively $20 \times 10^{6}$ and $160 \times 10^{6}$ and $80 \times 10^{6} \mathrm{kHz} / \mathrm{mm}^{2}$ for the two structures.

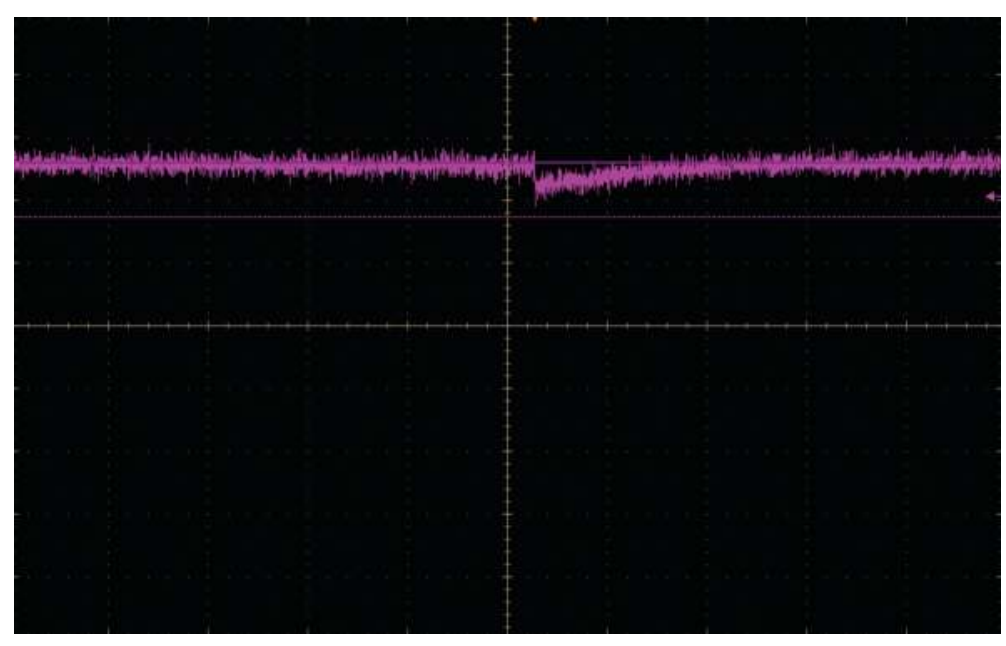

Figure 12. Structure I: signal corresponding to the thermal generation of electron/hole pairs within a $1 \mu$ s time window. 


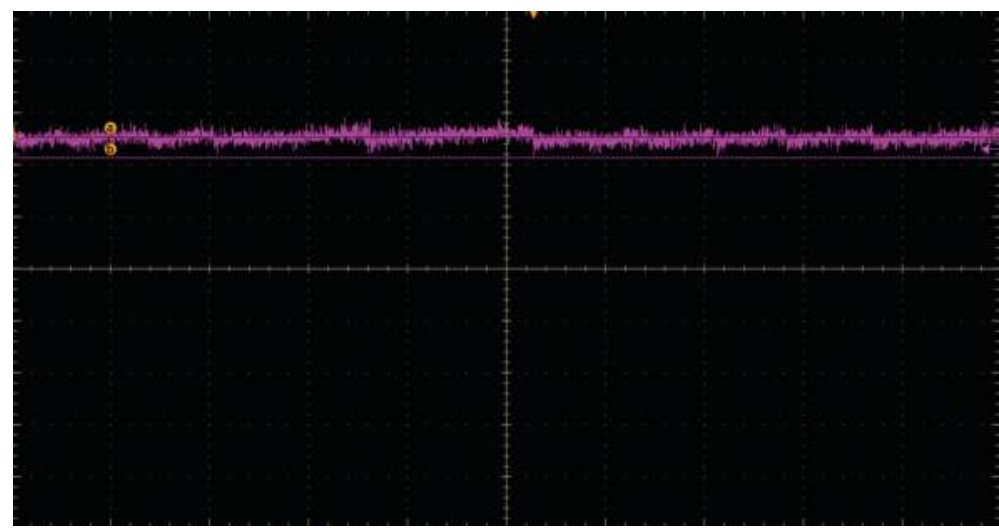

Figure 13. Structure II: signal corresponding to the thermal generation of electron/hole pairs within a $1 \mu$ s time window.

These measurements are well in agreement with the expectation from similar studies. Breakdown voltages ranging from 10 to $14 \mathrm{~V}$ are usually obtained at a scale lower than $180 \mathrm{~nm}$. This value depends on the doping of the standard CMOS wells, which ranges from $2 \times 10^{17}$ to $5 \times 10^{17}$. In comparison with custom technology the dark rate is few order of magnitudes higher. This is also a common feature of standard CMOS technology. Dark rate up to $100 \times 10^{5} \mathrm{kHz} / \mathrm{mm}^{2}$ was observed at $800 \mathrm{~nm}$ CMOS node [20]. The typical dark rate of 20$30 \times 10^{3} \mathrm{kHz} / \mathrm{mm}^{2}$ was observed at $180 \mathrm{~nm}$ CMOS node [55]. The results of the second structure show that the presence of the STI deteriorates the dark rate up to a factor 8 and confirms previous experiments [19-55]. These performances are worse that the state of the art SiPMs developed with custom technology, which obtain dark count rates as low as $30 \mathrm{kHz} / \mathrm{mm}^{2}$ and show that current CMOS technology needs improvements in order to fully accommodate the needs of SiPM sensor development $[15,17]$.

\section{The light response of the SiPM}

The response of the SiPM to light was studied only for a small prototype based on Structure I. The prototype consists on a $\mathrm{n}$ array of $5 \times 5$ microcells. A low photon flux source is characterized by a Poisson-distributed number of photons with a certain average value depending on the absolute intensity of the source and on the photon detection efficiency of the SiPM. The binomial contribution due to the not perfect photon detection efficiency is not perturbate the Poissonian nature of the detected photon distribution.

A precise test set up was prepared for the experimental study. Its key-component is a fast LED with wavelength $550 \mathrm{~nm}$. The light source is pulsed with a pulse width of $10 \mathrm{~ns}$. The time duration is chosen to be approximately ten times less than the recovery time of the SiPM. In order to exclude the electromagnetic pick up noise, the light pulses are delivered to the operation position in the light protected area by an optic fiber. The value of the low photon flux is controlled both electronically and by tuning its relative position with respect to the sensor. The alignment can be obtained with a precision of $0.1 \mathrm{~mm}$. Comparing the yeld of the 
developed SiPM with the yield of a commercially available SiPM with given characteristics we estimated that the photon detection efficiency of the developed SiPM is approximately $4 \%$. Such small value is expected, due to the isolation layers present on the active area of the device and the absence of an optical coupling window.

The SiPM output signal is amplified with a high quality current-voltage amplifier with gain 15 . Its charge is measured within an integration gate of $100 \mathrm{~ns}$ using the CAEN V1180 QDC in the VME Frame and stored in the control computer.

Figure 14 shows the experimental measured single photon spectrum. The black dots show clearly the specific form of the single photon spectrum. It consists of high resolved peaks correspondent to the number of photons distributed according to the Poisson statistics. Following the operation principle of the SiPM, every avalanche pixel detects one photon and gives as output the standard signal correspondent to a single photon. The common output of the SiPM is the analog sum of the signals from each avalanche pixel. In this condition, the first peak corresponds to 0 detected photons (electronic noise pedestal), the second one corresponds to 1 detected photon, the $(n+1)^{\text {th }}$ one to $n$ detected photons.

The number of peaks contains the information about the number of detected photons. The separation between two successive peaks has a constant magnitude and corresponds to the total number of electrons produced in the avalanches process. The area under each peak reflects the Poisson statistics of the photon detection in the SiPM structure.

The parameters of the expected Poisson distribution in the measured spectrum are estimated with a fit of the pedestal peak using the formula:

$$
\mu=\log \left(\frac{N}{N_{0}}\right)
$$

where $\mathrm{N}$ is the total number of events and $N_{0}$ is the number of events under the pedestal peak. We find that $\mu=4$ corresponds to the spectrum. As reported in the previous section, the SiPM

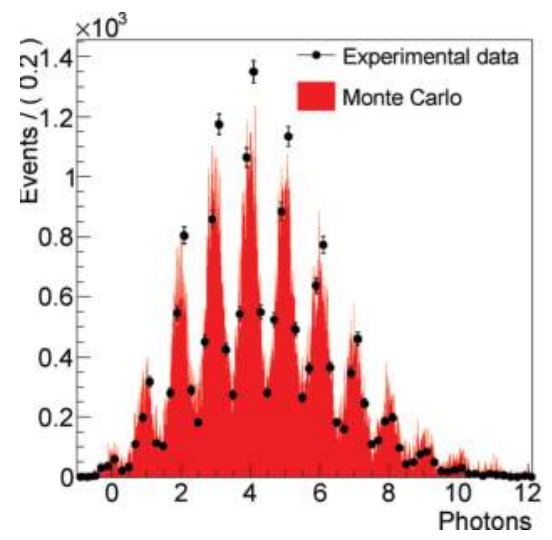

Figure 14. Measured spectrum corresponding to a low photon flux (black dots) and estimated Poisson distribution (filled area). 
sensor considered here exhibits a sizeable dark rate. We observe a deviation between theory and experimental data. In particular we report an excess of events at values higher than 2 detected photons. This shift in the observed distribution is due to dark rate and cross talk, which are particularly high in the produced SiPM sensor.

\section{Application of SiPM to digital positron emission tomography}

One of the most promising area of the application of the SiPM is nuclear medicine. Its small size, low bias voltage, simple read-out circuitry and fast response of the state I-II transition are opening the way of the development of a new concept of nuclear medicine diagnostic systems. Positron Emission Tomography (PET) is a functional nuclear medicine technique. A tracer is prepared marking specific ligands with beta + emitting isotopes. When ligands interact through the target molecular process, they are locally metabolized. The positrons emitted by the ligands annihilate with electrons within a mean free path of approximately $0.5 \mathrm{~mm}$ and as a result two photons with $511 \mathrm{keV}$ are emitted in opposite direction. Through the measurement of the photon directions it is possible to reconstruct the emission point and to have a detailed map of the activity of the ligands in the body.

A key-problem in PET is the technology used for the detection of the opposite photons and the reconstruction of their direction and energy. The basic block of modern PET is a detection system composed of a scintillator crystal read-out by a SiPM. When a $511 \mathrm{keV}$ photon deposits its energy in the crystal though photoelectric effect, scintillation light is produced and is detected by a SiPM. Due to the small size of the SiPM it is possible to design detector models which are more and more compact.

The LYSO/SiPM unit developed by the PETLab at the Huazhong University of Science and Technology consists of a $3.9 \times 3.9 \times 20 \mathrm{~mm}$ LySO crystal read out by a $3.0 \times 3.0 \mathrm{~mm} \mathrm{SiPM}$ by SensL (FM30035). A PET block is composed of an array of $6 \times 6$ LySO/SiPM elements, with pitch $4.2 \mathrm{~mm}$ and with $0.3 \mathrm{~mm}$ crystal gaps filled in with barium sulfate to guarantee the crystal optical coupling. LySO crystal and SiPM are aligned and coupled using epoxy optical adhesive $[63,64]$

On the basis of this pixelated block, a digital PET unit was developed. The key-component is here a digital electronic chain based on the Multi Voltage Threshold method. The basic idea of this new concept is that the digitalization of the signal produced when reading out the scintillation light from the crystal does not need a very high sampling. It is enough to sample 8 points corresponding to 4 thresholds. A double exponential fit to the signal shape through the interpolation of the sampled levels is enough to reconstruct the total integral of the signal, proportional to the number of detected scintillation photons and hence to the energy of the impinging gamma-ray, and the arrival time of the signal.

This novel digital PET system exhibited energy resolution between 10 and 15\%, time resolution of less than 200 ps, space resolution of approximately 1-2 mm. In particular it enabled a new concept of Positron Emission Tomography: the Plug\&Imaging (P\&I) technique. FPGA-only implementation allows one to achieve a high channel density with greatly simplified 
electronics. A complete software package for data acquisition, coincidence detection and image reconstruction (allowing a flexible overall geometry of the PET imager) has also been developed to support the P\&I feature and is shown on Figures $\mathbf{1 5}$ and $\mathbf{1 6}$. Such compact PET module can be in fact combined with other modules in different shapes and geometries, allowing a better match with the specific organs to be studied.

On the basis of this technology a full body digital PET scanner and a small animal PET scanner were successfully developed. However the modularity of the PET blocks allows a fast design of less standard approaches.

As an example of an easy combination of the P\&I elements, Positron Emission Tomography may be used in the correct determination of proton range in Proton Therapy. Protons interact with human tissue mainly with ionization, releasing their energy in the typical Bragg peak mechanism. However, nuclear interactions of protons with $\mathrm{O}, \mathrm{C}$ and $\mathrm{N}$ present in human tissues may have as results the production of neutrons associated with beta + emitters as ${ }^{15} \mathrm{O}$ and ${ }^{11} \mathrm{C} 11$. PET systems will be dedicated to the reconstruction of the position of such nuclear interaction. A comparison with Monte Carlo allows reconstructing the position of the Bragg peak from the space distribution of the nuclear interactions in the patient's body.

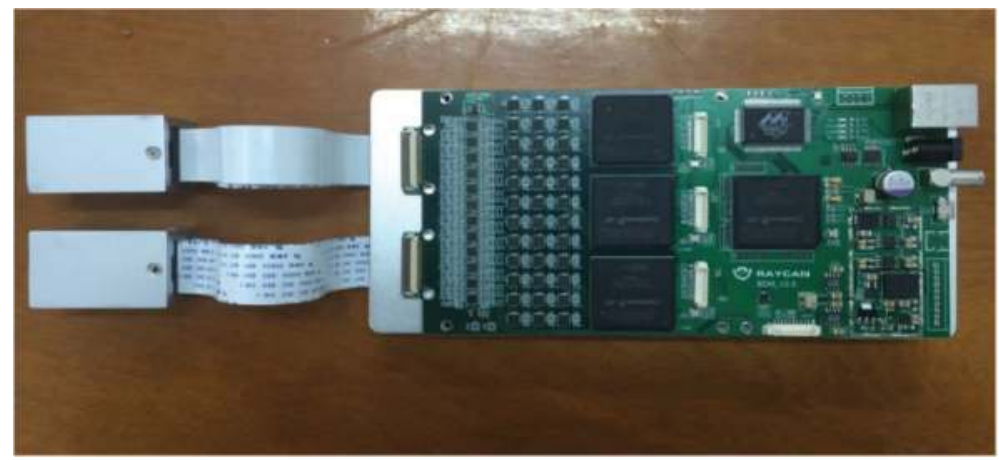

Figure 15. Plug \& Imaging PET detector. Two PET heads and the digital readout electronic boards are visible.

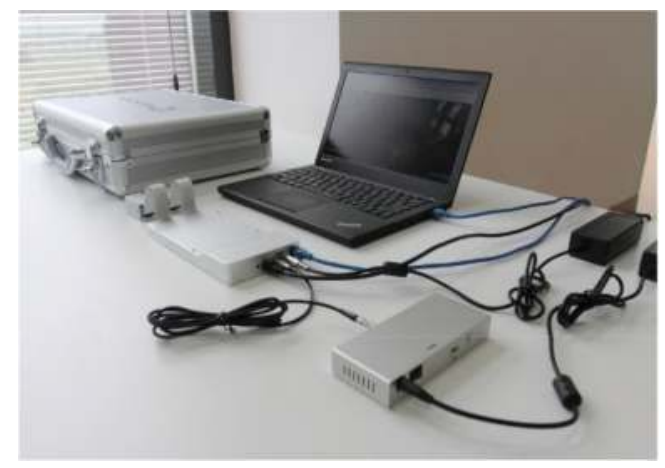

Figure 16. Plug \& Imaging PET detector: the mounted system with software chain. 


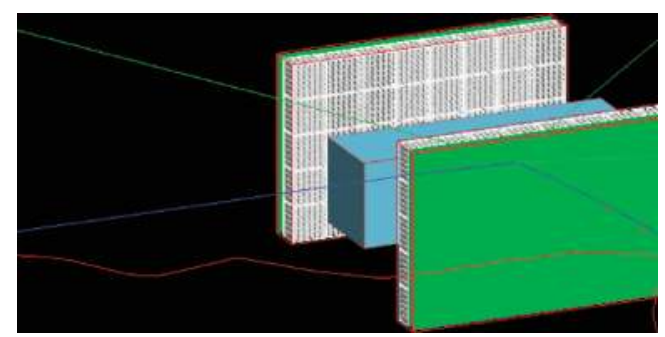

Figure 17. Simulation of a PET system for proton therapy monitoring and treatment.

Figure 17 shows the Monte Carlo of a PET system for Proton Therapy. It consists of two modules composed of 40 PET blocks, disposed on an array of $8 \times 5$. The total size of one head is $12.5 \times 20 \mathrm{~cm}$. Figure shows a typical event obtained with a proton beam energy of $160 \mathrm{MeV}$ in a water phantom. The nuclear reaction $\mathrm{p}+{ }^{16} \mathrm{O} \rightarrow{ }^{15} \mathrm{O}+\mathrm{p}+\mathrm{n}+$ gamma is followed by the $\beta+$ decay of the ${ }^{15} \mathrm{O} \rightarrow{ }^{15} \mathrm{~N}+\mathrm{e}^{+}+\mathrm{v}_{\varepsilon}{ }^{-}$. The positron annihilates into two gamma rays, generates then the PET signal. The design of the proton therapy monitoring system is particularly suited to the cure of brain cancer.

However the P\&I detection system based on the SiPM allows even more possibilities of dedicated PET scanners, as PET helmets for brain imaging. The technological challenges imposed by the development of this new kind of modular digital PET detection systems, together with the improvements in the medical diagnostic, such as the development of new ligands for the early detection of Alzheimer disease, will impose new benchmarks and new trends in modern Positron Emission Tomography and nuclear medicine.

One technological problem remains. The SiPM used in such new instrumentation for Nuclear Medicine are still obtained with custom technology. On the one hand, as we observed in the previous sections, the performances of mature custom technology is clearly better than the one achieved in standard CMOS. Nowadays also the price of mature technology is more attractive for PET producers. However, the possibility of the implementation of MVT digital electronics and SiPM sensor in a single chip offered by the CMOS implementation will significantly impact the compactness of the PET block realization, with a consequent miniaturization of the system and the possibility of even better performances of the P\&I method in terms of scalability, modularity and system space resolution.

\section{Conclusions}

The development of Silicon Photomultipliers within standard CMOS technology reveals a clear multi-disciplinary interest and matches the requirement that nowadays are coming also from the clinician's community. Digital electronics and digital information is in fact driving a change in the predictivity of diseases and the development of key-digital-technologies, as advanced CMOS SiPMs, will be fundamental in order to develop accurate instrumentation 
for the combination of techniques in Nuclear Medicine. The development of the P\&I platform is a clear example of such new paradigm for Positron Emission Tomography.

CMOS SiPMs still have space of improvement, which needs to be addressed. First the localized breakdown typical of SiPM structures could be corrected. However we found that only the SiPM structure with Shallow Trench Isolation placed far from the sensitive detector region exhibits a proper signal in correspondence to the production of an $\mathrm{e} / \mathrm{h}$ pair, the other devices being dominated by additional noise sources due to impurities dislocation on the border of the edges of the STI.

Even in this case the minimal achieved dark rate is approximately $10 \mathrm{MHz} / \mathrm{mm}^{2}$. This level is above the reached state of the art of current SiPM detectors produced in CMOS compatible processes with additional masks in the sensor region and suggests that the higher concentration of standard $180 \mathrm{~nm}$ CMOS processes is a limitation in the performances of the detector.

The photon detection efficiency obtainable in standard CMOS foundries is also very low. However, we observe that, although the standard $180 \mathrm{~nm}$ CMOS processes used to manufacture the APIX prototype does not allow us for an optimization of the optical window with a proper ARC technique, the manufactured structure I exhibits an excellent single photon spectrum, which is corresponding to a very good approximation to the Poisson statistics of the quantum state of detected photons.

These results shows that, although some improvements are needed with respect to concentration and STI technique, the standard CMOS technology is getting mature for a perspective, easy accessible, cost reductive SiPM optical detectors R\&D. This topic is so sensitive, that standard CMOS facilities are currently introducing new specific processes for APIX-like optical detectors with improved performance, allowing us for further steps consisting of the optimization of the technological parameters as well as of the optical performances, including ARC processes for improved photon detection efficiency.

\section{Acknowledgements}

This work was supported in part by the Natural Science Foundation of China (NSFC) Grant \#61425001, \#61210003, \#61604059, in part by the Natural Science Foundation of Hubei Province Grant \#2016CFA005, in part by National Key Scientific Instrument and Equipment Development Project of China \#2013YQ030923, in part by National Key Scientific Instrument and Equipment Development Project of Hubei Province \#2013BEC050.

\section{Author details}

Nicola D'Ascenzo and Qingguo Xie*

*Address all correspondence to: qgxie@hust.edu.cn

Huazhong University of Science and Technology, Wuhan, China 


\section{References}

[1] Dascenzo N, Saveliev V. The new photodetectors for high energy physics and nuclear medicine. In: Shi J-W, editor. Photodiodes. Croatia: Intech; 2011. pp. 261-284

[2] D'Auria V, Lee N, Amri T, Fabre C, Laurat J. Quantum decoherence of single photon counters. Physical Review Letters. 2011;107(5):050504. DOI: 10.1103/PhysRevLett.107.050504

[3] Achilles D, Silberhorn C, Silwa C, Banaszek K, Walmsley A. Fiberassisted detection with photon number resolution. Optics Letters. 2003;28(23):2387-2389. DOI: 10.1364/OL.28.002387

[4] Gasanov A, Golovin V, Sadigov ZY, Yusipov NY. Avalanche photodetector based on metal-resistive-layer semiconductor structures. Pisma V Zhurnal Tekhnicheskoi Fiziki. 1988;14:706-709

[5] Shushakov DA, Shubin VE. New solid state photomultiplier. In: Photonics West 95; International Society for Optics and Photonics; 1995. pp. 544-554

[6] Sadygov Z. Avalanche detector. Russian Patent. 1996; RU 2102820

[7] Golovin M, Akindinov A, Grigorev E, Martemyanov A, Polozov P. New results on MRS APD. Nuclear Instruments and Methods in Physics Research. 1997;387:231-234

[8] Saveliev V, Golovin V. Silicon avalanche photodiodes on the base of metal-resistor-semiconductor (MRS) structures. Nuclear Instruments and Methods in Physics Research. 2000;442:223-229

[9] Buzhan P, Dolgoshein B, Ilyin A, Kantser V, Kaplin V, Karakash A, Pleshko A, Popova E, Smirnov S, Volkov Y. An advanced study of silicon photomultiplier. ICFA Instrumentation Bulletin. 2001;21:28

[10] Piemonte C, Battiston R, Boscardin M, Dalla Betta G-F, Del Guerra A, Dinu N, Pozza A, Zorzi N. Characterization of the first prototypes of silicon photomultiplier fabricated at ITC IRST. IEEE Transactions on Nuclear Science. 2007;54:236-244

[11] Stewart A, Saveliev V, Bellis S, Herbert DJ, Huges P, Jackson J. Performance of a $1 \mathrm{~mm}^{2}$ silicon photomultiplier. IEEE Journal of Quantum Electronics. 2008;44:157-164

[12] Ghassemi A, Sato K, Kobayashi K. Hamamatsu Technical Note 2017; KAPD9005E01

[13] Cova S, Lacaita A, Ghioni M, Ripamonti G, Louis T. 20 ps timing resolution with singlephoton avalanche diodes. The Review of Scientific Instruments. 1989;60:1104-1110

[14] Villa F, Bronzi D, Zou Y, Scarcella C, Boso G, Tisa S, Tosi A, Zappa F, Durini D, Weyers S. CMOS SPADs with up to $500 \mu \mathrm{m}$ diameter and 55\% detection efficiency at $420 \mathrm{~nm}$. Journal of Modern Optics. 2014;61:102-115

[15] Schwinger A, Brockherde W, Hosticka BJ, Vogt H. CMOSSiPM with integrated amplifier. Proceedings of SPIE. Feb. 2017;10100:101001A. DOI: 10.1117/12.2252516

[16] Della Rocca FM, Nedbal J, Tyndall D, Kristajic N, Day-Uei Li D, Ameer-Beg SM, Henderson RK. Real-time fluorescence lifetime actuation for cell sorting using a CMOS SPAD silicon photomultiplier. Optics Letters. Feb. 2016;41(4):673-676. DOI: 10.1364/OL.41.000673 
[17] Zou Y, Villa F, Bronzi D, Tisa S, Tosi A, Zappa F. Planar CMOS analog SiPMs: Design, modeling, and characteriza- tion. Journal of Modern Optics. May 2015;62(20):1693-1702. DOI: $10.1080 / 09500340.2015 .1049572$

[18] Bérubé BL, Rhéaume VP, Parent S, Maurais L, Therrien AC, Charette PG, Charlebois SA, Fontaine R, Pratte JF. Implementation study of single photon avalanche diodes (spad) in $0.8 \mu \mathrm{m}$ HV CMOS technology. IEEE Transactions on Nuclear Science. Jun. 2015;62(3):710718. DOI: $10.1109 /$ TNS.2015.2424852

[19] D'Ascenzo N, Zhang X, Xie Q. Application of CMOS technology to silicon photomultiplier sensors. Sensors. Sep. 2017;17:2204

[20] D'Ascenzo N, Brockherde W, Dreiner S, Schwinger A, Schmidt A, Xie Q. Design and characterization of a silicon photomultiplier in 0.35 um CMOS. IEEE Journal of the Electron Devices Society. Accepted Nov. 2017. DOI: 10.1109/JEDS.2017.2771145

[21] Niclass C, Rochas A, Besse P-A, Charbon E. Toward a 3-D camera based on single photon avalanche diodes. IEEE Journal of Selected Topics in Quantum Electronics. 2004;10:796-802

[22] Rochas A, Gosch M, Serov A, Besse P, Popovic R, Lasser T, Rigler R. First fully integrated 2-D array of single-photon detectors in standard CMPS technology. IEEE Photonics Technology Letters. 2003;15:963-965

[23] Tisa S, Zappa F, Labanca I. In On-chip detection and counting of single-photons, Electron Devices Meeting, 2005. IEDM Technical Digest. IEEE International: IEEE; 2005. pp. 815-818

[24] Stoppa D, Pancheri L, Scandiuzzo M, Gonzo L, Dalla Betta G-F, Simoni A. A cmos 3-d imager based on single photon avalanche diode. IEEE Transactions on Circuits and Systems. 2007;54:4-12

[25] Niclass C, Rochas A, Besse P-A, Charbon E. Design and characterization of a CMOS 3-D image sensor based on single photon avalanche diodes. IEEE Journal of Solid-State Circuits. 2005;40:1847-1854

[26] Stoppa D, Pancheri L, Scandiuzzo M, Malfatti M, Pedretti G, Gonzo L. In a singlephoton-avalanche-diode 3D imager, Solid-State Circuits Conference, 2005. ESSCIRC 2005. Proceedings of the 31st European: IEEE; 2005. pp. 487-490

[27] Rochas A, Gani M, Furrer B, Besse P, Popovic R, Ribordy G, Gisin N. Single photon detector fabricated in a complementary metal-oxide-semiconductor high-voltage technology. Review of Scientific Instruments. 2003;74:3263-3270

[28] Bérubé B-L, Rhéaume V-P, Parent S, Maurais L, Therrien AC, Charette PG, Charlebois SA, Fontaine R, Pratte J-F. Implementation study of single photon avalanche diodes (spad) in $0.8 \mu \mathrm{m}$ HV CMOS technology. IEEE Transactions on Nuclear Science. 2015;62:710-718

[29] Pancheri L, Stoppa D. In low-noise CMOS single-photon avalanche diodes with 32 ns dead time, Solid State Device Research Conference, 2007. ESSDERC 2007. 37th European; IEEE; 2007. pp. 362-365

[30] Habib MHU, McFarlane N. In a perimeter gated single photon avalanche diode based silicon photomultiplier as optical detector, Circuits and Systems (MWSCAS), 2015 IEEE 58th International Midwest Symposium on: IEEE: 2015. pp. 1-4 
[31] Gu J, Habib MHU, McFarlane N. Perimeter gated single photon avalanche diodes: An information theoretic assessment. IEEE Photonics Technology Letters. 2016;28:701-704

[32] Niclass C, Sergio M, Charbon E. In a single photon avalanche diode array fabricated in $0.35 \mu \mathrm{m}$ CMOS and based on an event-driven readout for TCSPC experiments. Proceedings Volume 6372, Advanced Photon Counting Techniques; 63720S, 2006

[33] Niclass C, Favi C, Kluter T, Monnier F, Charbon E. Single-photon synchronous detection. IEEE Journal of Solid-State Circuits. 2009;44:1977-1989

[34] Tisa S, Guerrieri F, Tosi A, Zappa F. In 100 kframe/s 8 bit monolithic single-photon imagers, Solid-State Device Research Conference. ESSDERC 2008. 38th European, 2008; IEEE; 2008. pp. 274-277

[35] Tisa S, Guerrieri F, Zappa F. Variable-load quenching circuit for single-photon avalanche diodes. Optics Express. 2008;16:2232-2244

[36] Stoppa D, Mosconi D, Pancheri L, Gonzo L. Single-photon avalanche diode cmos sensor for time-resolved fluorescence measurements. IEEE Sensors Journal. 2009;9:1084-1090

[37] Arbat A, Trenado J, Gascon D, Vilá A, Comerma A, Garrido L, Diéguez A. High voltage vs. high integration: A comparison between CMOS technologies for SPAD cameras. Proceedings of SPIE Optics and Photonics. 2010;2010, 7780:77801G

[38] Niclass C, Favi C, Kluter T, Gersbach M, Charbon E. A $128 \times 128$ single-photon image sensor with column-level 10-bit time-to-digital converter array. IEEE Journal of SolidState Circuits. 2008;43:2977-2989

[39] Vilella E, Alonso O, Montiel A, Vilá A, Diéguez A. A low-noise time-gated single-photon detector in a HV-CMOS technology for triggered imaging. Sensors and Actuators A: Physical. 2013;201:342-351

[40] Nissinen I, Nissinen J, Lansman A, Hallman L, Kilpel AA, Kostamovaara J, Kogler, M, Aikio M, Tenhunen J. In a sub-ns time-gated CMOS single photon avalanche diode detector for Raman spectroscopy, Solid-State Device Research Conference (ESSDERC), 2011 Proceedings of the European: IEEE; 2011. pp. 375-378

[41] Schwinger A, Brockherdea W, Hostickaa BJ, Vogta H. In CMOS SiPM with integrated amplifier. Proceedings of SPIE. 2017:101001A-1101001

[42] Zou Y, Villa F, Bronzi D, Tisa S, Tosi A, Zappa F. Planar CMOS analog SiPMs: Design, modeling, and characterization. Journal of Modern Optics. 2015;62:1693-1702

[43] Vilella E, Diéguez A. A gated single-photon avalanche diode array fabricated in a conventional CMOS process for triggered systems. Sensors and Actuators A: Physical. 2012; 186:163-168

[44] Jradi K, Pellion D, Ginhac D. Design, characterization and analysis of a $0.35 \mu \mathrm{m}$ CMOS SPAD. Sensors. 2014;14:22773-22784 
[45] Finkelstein H, Hsu MJ, Esener SC. Sti-bounded single-photon avalanche diode in a deepsubmicrometer cmos technology. IEEE Electron Device Letters. 2006;27:887-889

[46] Faramarzpour N, Deen MJ, Shirani S, Fang Q. Fully integrated single photon avalanche diode detector in standard cmos $0.18-\mu \mathrm{m}$ technology. IEEE Transactions on Electron Devices. 2008;55:760-767

[47] Vornicu I, Bandi FN, Carmona-Galán R, Rodríguez-Vázquez Á. A CMOS digital SiPM with focal-plane light-spot statistics for DOI computation. IEEE Sensors Journal. 2017; 17:632-643

[48] Pancheri L, Stoppa D. In low-noise single photon avalanche diodes in $0.15 \mu \mathrm{m}$ CMOS technology, Solid-State Device Research Conference (ESSDERC). Proceedings of the European, 2011: IEEE; 2011. pp. 179-182

[49] Richardson JA, Grant LA, Henderson RK. Low dark count single-photon avalanche diode structure compatible with standard nanometer scale cmos technology. IEEE Photonics Technology Letters. 2009;21:1020-1022

[50] Gersbach M, Maruyama Y, Trimananda R, Fishburn MW, Stoppa D, Richardson JA, Walker R, Henderson R, Charbon E. A time-resolved, low-noise single-photon image sensor fabricated in deep-submicron CMOS technology. IEEE Journal of Solid-State Circuits. 2012;47:1394-1407

[51] Henderson RK, Richardson J, Grant L. Reduction of Band-to-Band Tunneling in DeepSubmicron cmos Single Photon Avalanche Photodiodes. Bergen, Norway: International Image Sensor Workshop (IISW 2009); 2009. pp. 26-28

[52] Karami MA, Gersbach M, Yoon H-J, Charbon E. A new single-photon avalanche diode in 90nm standard CMOS technology. Optics Express. 2010;18:22158-22166

[53] Webster EA, Richardson JA, Grant LA, Renshaw D, Henderson RK. A single-photon avalanche diode in 90-nm CMOS imaging technology with $44 \%$ photon detection efficiency at $690 \mathrm{~nm}$. IEEE Electron Device Letters. 2012;33:694-696

[54] Herbert DJ, Moehrs S, D'Ascenzo N, Del Guerra A. The silicon photomultiplier for application to high-resolution positron emission tomography. Nuclear Instruments and Methods. 2007;573:84-87

[55] Colletti PM. SNMM: Highlights lecture: General nuclear medicine. JNM. 2017;58:11N-15N

[56] Shockley W, Read WT. Statistics of the recombination of holes and electrons. Physics Review. 1952;87:835

[57] Selberherr S. Analysis and Simulation of Semiconductor Devices. Wien, New York: Springer-Verlag; 1984

[58] Izhaky N, Morse MT, Koehl S, Cohen O, Rubin D, Barkai A, Sarid G, Cohen R, Paniccia MJ. Development of CMOS-compatible integrated silicon photonics devices. IEEE Journal of Selected Topics in Quantum Electronics. 2006;12:1688-1698 
[59] Lee M-J, Rucker H, Choi W-Y. Effects of guard-ring structures on the performance of silicon avalanche photodetectors fabricated with standard CMOS technology. IEEE Electron Device Letters. 2012;33:80-82

[60] Lee M-J, Choi W-Y. Effects of parasitic resistance on the performance of silicon avalanche photodetectors in standard CMOS technology. IEEE Electron Device Letters. 2016;37:60-63

[61] Sul W-S, Oh J-H, Lee C-H, Cho G-S, Lee W-G, Kim S-D, Rhee J-K. Guard-ring structures for silicon photomultipliers. IEEE Electron Device Letters. 2010;31:41-43

[62] MINICIRCUITS. www.minicircuits.com

[63] Xie Q, Chen Y, Yuanbao Z. Implementation of LYSO/PSPMT block detector with all digital DAQ system. IEEE Transactions on Nuclear Science. 2013;60(3):1487-1494

[64] Xie Q, Kao C, Byrun K. Characterization of Silicon Photomultipliers for PET imaging, in 2016 IEEE Nuclear science conference records. Vol. 1-6; 2008. pp. 1199-1203 